\title{
Ten Principles for More Conservative, Care-Full Diagnosis
}

Gordon D. Schiff, MD; Stephen A. Martin, MD, EdM; David H. Eidelman, MD; Lynn A. Volk, MHS; Elise Ruan, BS; Christine Cassel, MD; William Galanter, MD; Mark Johnson, MD, MS; Annemarie Jutel, PhD; Kurt Kroenke, MD; Bruce L. Lambert, PhD; Joel Lexchin, MSc, MD; Sara Myers, BA; Alexa Miller, MA; Stuart Mushlin, MD; Lisa Sanders, MD; Aziz Sheikh, MD Many spotlights currently illuminate the challenges associated with medical diagnosis. The National Academy of Medicine estimates that all patients will experience 1 serious diagnostic error during their lifetime, and diagnostic errors are now the leading cause of medical malpractice claims $(1,2)$. To avoid missing diagnoses, clinicians often order imaging and/or laboratory studies and initiate specialist referrals. However, physicians and patients are also urged to use fewer tests; nearly every U.S. medical specialty and 20 countries worldwide have initiated Choosing Wisely campaigns (3). Evidence increasingly shows that indiscriminate diagnostic testing and referrals often fail to provide definitive explanations or improve outcomes and at times are more harmful than beneficial.

Balancing underdiagnosis (missing or delaying important diagnoses) and wasteful, harmful overdiagnosis (labeling patients with diseases that may never cause suffering or death) is often portrayed as the need "to keep the pendulum from swinging too far in either direction" (4). Rather than framing the problem as a simple, linear tradeoff, we believe it must be more fundamentally conceptualized as 2 sides of the same coin unified by the need for more cautious and careful approaches.

This is the author's manuscript of the work published in final form as:

Schiff, G. D., Martin, S. A., Eidelman, D. H., Volk, L. A., Ruan, E., Cassel, C., Galanter, W., Johnson, M., Jutel, A., Kroenke, K., Lambert, B. L., Lexchin, J., Myers, S., Miller, A., Mushlin, S., Sanders, L., \& Sheikh, A. (2018). Ten Principles for More Conservative, Care-Full Diagnosis. Annals of Internal Medicine, 169(9), 643-645. https://doi.org/10.7326/M18-1468 
We assembled a diverse group of clinicians, educators, and health policy and communication experts to create recommendations to support improved approaches to clinical care and health policy (www.patientsafetyresearch.org/Schiff_Ten_Principles_Conservative_Diagnosis.pdf). Building on our previous conservative principles of medication prescribing (5), we developed 10 overarching principles based on core attributes of care (good communication, trusting relationships, and continuity of care) and key patient safety lessons (awareness of pitfalls, safety nets to mitigate harm, and a culture that facilitates learning/avoiding blame) that go beyond current test-by-test recommendations.

\section{Promoting Enhanced Caring and Listening}

Patients come to clinicians seeking explanations for their symptoms. Clinicians often rely on laboratory and imaging studies and specialist referrals to rule out serious diagnoses and identify patients who could benefit from particular treatments. However, this approach rests on the questionable assumptions that testing is key to making an accurate diagnosis, an exact diagnosis is always available and needed to select therapies, and ordering tests best shows that clinicians are taking patients' concerns seriously.

Medicine currently shortchanges the patient history and physical examination, even though carefully listening to and observing patients over time often provide more valuable information than multiple radiologic or chemical tests. We must stop equating testing with caring and thoroughness and instead emphasize respectful listening, examination, follow-up, and collaboration with the patient to "coproduce" diagnoses $(1,6)$.

\section{Developing a New Science of Uncertainty}


As precision medicine becomes a major preoccupation, appreciation of the pervasiveness of uncertainty in medicine has paradoxically increased (7). We need to develop a new science and praxis of diagnostic uncertainty that acknowledges complex biological and social systems and serves as a starting point for more modest, reflective, and conservative practice. Doing so requires acknowledging widespread uncertainty, better operationalizing follow-up, and communicating honestly about uncertainty.

\section{Rethinking Symptoms}

Up to one half of symptoms defy definitive medical diagnosis. Further, many symptoms are selflimiting: $75 \%$ to $80 \%$ of symptoms improve over 4 to 12 weeks, usually regardless of medical intervention (8). Some patients meet criteria for depression, anxiety, or somatoform illnesses, yet these diagnoses are overlooked in two thirds of patients. Visits for "medically unexplained symptoms" currently represent the fastest-growing type of medical encounter. Caring for these patients can be frustrating, leading clinicians to be dismissive or stigmatizing. We need to move away from exhaustively trying to rule out multiple rare diseases and then labeling patients' symptoms as nonorganic, toward more helpful and supportive approaches.

\section{Maximizing Continuity and Trust}

Continuity is the foundation of judicious clinical practice. Without knowledgeable, trusting relationships, clinicians must often resort to defensive, inadequately informed, and costly styles of practice. Health systems that maximize relational and informational continuity perform better and cost less (9), and patients value having clinicians who know them well. Financial incentives 
can undermine long-term, trusting relationships. If clinicians are incentivized to withhold tests, patients may find trusting "watch-and-wait" recommendations difficult (3).

\section{Taming and Taking Time}

Time is the currency of clinical care. Although few clinicians would disagree in principle with the conservative diagnosis practices that we advocate, many argue that they simply do not have time for prolonged discussions about uncertainty, exploration of symptoms in greater detail, or comprehensive follow-up. Time is a powerful incubator for diagnosis. Conservative diagnosis requires carefully and skillfully weighing information as it evolves. Having adequate time to listen, observe, discuss, and reflect is a decisive factor that separates good diagnosis from underand overdiagnosis. Practical strategies include redesigning care to optimize the roles of other team members and reengineering electronic health records and follow-up systems to support watchful waiting, a fundamental pillar of conservative diagnosis and an antidote to the unwatchful neglect that patients fear (10).

\section{Linking Diagnosis to Treatment}

Diagnosis needs to stand less alone and more arm in arm with treatment. The value of diagnosis is greater in conditions with effective, specific, or urgent treatments and more limited if no therapy is available, a diagnosis is not needed to select among treatment options, and/or treatment can be safely deferred. Diagnosing conditions for which patients have no interest in being treated (for example, chemotherapy and surgery) may be unwarranted and disrespectful.

\section{Ordering and Interpreting Tests More Thoughtfully}


Conservative diagnosis is not just saying "no" to tests or the patients requesting them. Rather, it is about more intelligently selecting, timing, sequencing, interpreting, and weighing the marginal benefits of tests. Few appreciate the biases and lack of rigor involved in evaluating new diagnostic tests, which are not subject to the same evidence and regulatory standards as medications. We also often do not fully consider the potential harms of testing (Table).

\section{Safety Nets: Incorporating Lessons From Diagnostic Errors}

Recent attention given to diagnostic errors might seem to argue for more aggressive defensive medicine to rule out myriad diagnoses lest they be missed and labeled as errors and delays. However, additional testing does not necessarily result in answers that patients and clinicians seek. Being aware of potential diagnostic errors can help avoid pitfalls and build safety nets and systems to protect against known errors.

\section{Addressing Cancer: Fears and Challenges}

Patients understandably fear missed cases of cancer. Almost any symptom can be due to cancer. Clinicians and the media have long promoted early diagnosis, but serious controversies surround efforts to screen for and diagnose most types of cancer. These issues are complex. Furthermore, data are often inconclusive or conflicting, particularly considering such issues as lead-time bias; overdiagnosis of cancer that is incidentally discovered but best left untreated; false-positive and false-negative test results; uncertainties about the value of treatment; and questions about the marginal benefit of early treatment. We need to help patients understand the toll imposed by false-positive results and overdiagnosed cancer to appreciate the need to strike a balance between treating the few with harmful cancer and avoiding harm to the many without it. 


\section{Diagnostic Stewardship: Transforming the Role of Specialists and Emergency Department}

\section{Clinicians}

Implicit in conservative diagnosis is minimizing indiscriminate use of specialty referrals and emergency departments. However, both specialists and emergency department clinicians can positively contribute by leveraging their knowledge and playing stewardship roles. Specialists can provide guidance when testing or referring is not needed and offer safety nets (such as triage electronic consultations/second opinions and guidelines) to conservatively assess and reassure patients. Emergency department clinicians can work with primary care clinicians to help reduce unnecessary emergency department visits while helping to expedite truly urgent evaluations.

\section{Conclusion}

Achieving more judicious diagnosis mandates policy support to redesign care at both the individual patient and system levels. Practical safety nets can protect the safety and quality of diagnosis and promote more conservative practice (www.patientsafetyresearch.org/Schiff_Ten_Principles_Conservative_Diagnosis.pdf). 


\section{Authorship and Disclosures}

Brigham and Women's Hospital and Harvard Medical School, Boston, Massachusetts (G.D.S., M.J.)

University of Massachusetts Medical School, Worcester, Massachusetts (S.A.M.)

McGill University, Montreal, Quebec, Canada (D.H.E.)

Brigham and Women's Hospital, Boston, Massachusetts, and Partners HealthCare, Somerville, Massachusetts (L.A.V., S.M.)

Brigham and Women's Hospital and Tufts University School of Medicine, Boston, Massachusetts, and Partners HealthCare, Somerville, Massachusetts (E.R.)

Kaiser Permanente School of Medicine, Pasadena, California (C.C.)

University of Illinois, Chicago, Chicago, Illinois (W.G.)

Harvard Medical School, Boston, Massachusetts; Victoria University of Wellington, Wellington, New Zealand (A.J.)

Indiana University, Indianapolis, Indiana (K.K.)

Northwestern University, Chicago, Illinois (B.L.L.)

York University, Toronto, Ontario, Canada (J.L.)

ArtsPractica, Guilford, Connecticut (A.M.)

Brigham Circle Medical Associates, Boston, Massachusetts (S.M.)

Yale University School of Medicine, New Haven, Connecticut (L.S.)

The University of Edinburgh, Edinburgh, United Kingdom (A.S.)

Note: Drs. Martin, Eidelman, Cassel, Galanter, Johnson, Jutel, Kroenke, Lambert, Lexchin, Mushlin, Sanders, and Sheikh and Ms. Myers are members of an expert panel assembled for collaborative development of conservative diagnosis principles.

Disclaimer: The funding source had no role in the design or conduct of the study; collection, analysis, or interpretation of the data; or preparation or review of the manuscript. The findings and conclusions in this commentary are those of the authors and do not necessarily represent the official position of the Gordon and Betty Moore Foundation.

Acknowledgment: The authors thank Ami Karlage for editorial support and Andrea Lim for manuscript support.

Grant Support: From the Gordon and Betty Moore Foundation.

Disclosures: Disclosures can be viewed at www.acponline.org/authors/icmje/ConflictOfInterestForms.do?msNum=M18-1468.

Corresponding Author: Gordon D. Schiff, MD, Brigham and Women's Hospital, 1620 Tremont Street, 3rd Floor, Room 03-02-2N, Boston, MA 02120; e-mail, gschiff@partners.org.

Current Author Addresses: Dr. Schiff: Brigham and Women's Hospital, 1620 Tremont Street, 3rd Floor, Room 03-02-2N, Boston, MA 02120.

Dr. Martin: Barre Family Health Center, 151 Worcester Road, Barre, MA 01005. 
Dr. Eidelman: McGill University, 3605 Rue de la Montagne, Montreal, Quebec H3G 2M1, Canada.

Ms. Volk: Partners HealthCare, 399 Revolution Drive, Somerville, MA 02145.

Ms. Ruan: Tufts University, 2 Hawthorne Place, Boston, MA 02114.

Dr. Cassel: 64 Lodge Trail, Santa Fe, NM 87506.

Dr. Galanter: Section of Academic Internal Medicine, 840 S. Wood, 440D, Chicago, IL 60612.

Dr. Johnson: Department of Medicine, Mount Auburn Hospital, 330 Mount Auburn Street, Cambridge, MA 02138.

Prof. Jutel: Victoria University of Wellington, PO Box 600, Kelburn, Wellington 6140, New

Zealand.

Dr. Kroenke: Regenstrief Institute, 1101 West 10th Street, RF 221, Indianapolis, IN 46202.

Dr. Lambert: Northwestern University, 710 N. Lake Shore Drive, 15th Floor, Chicago, IL 60611.

Dr. Lexchin: York University, 4700 Keele Street, Toronto, Ontario M3J 1P3, Canada.

Ms. Myers: 2783 Lancashire Road, Apartment 10, Cleveland, OH 44106.

Ms. Miller: 201 Dromara Road, Guilford, CT 06437.

Dr. Mushlin: Brigham Circle Medical Associates, 75 Francis Street, Boston, MA 02115.

Dr. Sanders: St. Raphael's Hospital, 1450 Chapel Street, Room M423E, New Haven, CT 06511.

Dr. Sheikh: Usher Institute of Population Health Sciences and Informatics, The University of

Edinburgh, Teviot Place, Edinburgh EH8 9AG, United Kingdom.

Author Contributions: Conception and design: G.D. Schiff, S.A. Martin, D.H. Eidelman, L.A. Volk, E. Ruan, S. Myers.

Drafting of the article: G.D. Schiff, S.A. Martin, D.H. Eidelman, L.A. Volk, E. Ruan, C. Cassel, W. Galanter, M. Johnson, A. Jutel, K. Kroenke, B.L. Lambert, J. Lexchin, S. Myers, A. Miller, S. Mushlin, L. Sanders, A. Sheikh.

Critical revision of the article for important intellectual content: G.D. Schiff, S.A. Martin, D.H. Eidelman, L.A. Volk, E. Ruan, C. Cassel, W. Galanter, M. Johnson, A. Jutel, K. Kroenke, B.L. Lambert, J. Lexchin, S. Myers, A. Miller, S. Mushlin, L. Sanders, A. Sheikh.

Final approval of the article: G.D. Schiff, S.A. Martin, D.H. Eidelman, L.A. Volk, E. Ruan, C. Cassel, W. Galanter, M. Johnson, A. Jutel, K. Kroenke, B.L. Lambert, J. Lexchin, S. Myers, A. Miller, S. Mushlin, L. Sanders, A. Sheikh.

Obtaining of funding: G.D. Schiff, L.A. Volk.

Administrative, technical, or logistic support: L.A. Volk, E. Ruan, S. Myers. 


\section{References}

1. Balogh E, Miller BT, Ball J, eds. Improving Diagnosis in Health Care. Washington, DC: National Academies Pr; 2015.

2. Schiff GD, Puopolo AL, Huben-Kearney A, Yu W, Keohane C, McDonough P, et al. Primary care closed claims experience of Mas-sachusetts malpractice insurers. JAMA Intern Med. 2013;173: 2063-8. [PMID: 24081145] doi:10.1001/jamainternmed.2013.11070

3. Levinson W, Kallewaard M, Bhatia RS, Wolfson D, Shortt S, Kerr EA; Choosing Wisely International Working Group. "Choosing Wisely": a growing international campaign. BMJ Qual Saf. 2015;24: 167-74. [PMID: 25552584] doi:10.1136/bmjqs-2014-003821

4. Landro L. A medical detective story: why doctors make diagnostic errors. The Wall Street Journal. 26 September 2015 Accessed at www.wsj.com/articles/a-medical-detective-storywhy-doctors-make-diagnostic-errors-1443295859 on 7 September 2018.

5. Schiff GD, Galanter WL, Duhig J, Lodolce AE, Koronkowski MJ, Lambert BL. Principles of conservative prescribing. Arch Intern Med. 2011;171:1433-40. [PMID: 21670331] doi:10.1001/archinternmed .2011.256

6. Hart JT. Clinical and economic consequences of patients as producers. J Public Health Med. 1995; 17:383-6. [PMID: 8639335]

7. Simpkin AL, Schwartzstein RM. Tolerating uncertainty-the next medical revolution? N Engl J Med. 2016;375:1713-5. [PMID: 27806221]

8. Kroenke K. A practical and evidence-based approach to common symptoms: a narrative review. Ann Intern Med. 2014;161:579-86.[PMID: 25329205] doi:10.7326/M14-0461

9. Starfield B, Shi L, Macinko J. Contribution of primary care to health systems and health. Milbank Q. 2005;83:457-502. [PMID: 16202000]

10. Schiff GD. Minimizing diagnostic error: the importance of follow-up and feedback. Am J Med. 2008;121:S38-42. [PMID: 18440354] doi:10.1016/j.amjmed.2008.02.004 
Table. Potential Harms from Diagnostic Testing*

\begin{tabular}{|c|}
\hline Direct Harm \\
\hline Complications from invasive tests \\
\hline Unstable patients leaving more protected environments to undergo tests \\
\hline Delays in initiation of urgent treatment while waiting for tests and results \\
\hline Adverse reactions (e.g., renal toxicity and anaphylaxis) from contrast or other diagnostic agents \\
\hline $\begin{array}{l}\text { Local complications from phlebotomy and catheter access (e.g., hematoma, contamination, pain from } \\
\text { multiple venipuncture sticks, and wounds) and loss of future venous access }\end{array}$ \\
\hline Imaging-associated cancer and other harms from radiation \\
\hline Downstream Harm \\
\hline $\begin{array}{l}\text { Harm from further work-up and treatment of false-positive test results (especially failure to account } \\
\text { for the poor predictive value of positive results in the context of low prior probability) }\end{array}$ \\
\hline $\begin{array}{l}\text { Harm from treatment caused by overdiagnosis (i.e., diagnosis of conditions that, although correctly } \\
\text { diagnosed, would never have caused harm or required treatment) }\end{array}$ \\
\hline False reassurance (i.e., complacency or failure to treat as a result of a false-negative result) \\
\hline Harm from additional testing, such as cascades after an initial false-positive result or overdiagnosis \\
\hline Conveying a message to patients that promotes a culture of indiscriminate testing \\
\hline Treatment of asymptomatic risk factors with interventions that cause \\
\hline Harm Intrinsic to Making a Diagnosis \\
\hline Stigmatizing labels that may outweigh any benefits of the diagnosis for a patient \\
\hline Anxiety from diagnoses that would not otherwise have been discovered or treated \\
\hline $\begin{array}{l}\text { Increased burden of illness (e.g., more medication regimens, appointments, and procedures and lost } \\
\text { work time and greater family burden) }\end{array}$ \\
\hline $\begin{array}{l}\text { Distraction of clinicians' and patients' attention from more beneficial diagnostic activities (e.g., } \\
\text { obtaining a better history and serial examinations) and treatment }\end{array}$ \\
\hline
\end{tabular}

* The risk for these adverse effects may be more or less frequent de-pending on the test, patient, or context but need to be recognized, weighed, and minimized when ordering and performing diagnostic tests. 